\title{
tDCS-Induced Modulation of GABA Levels and Resting-State Functional Connectivity in Older Adults
}

\author{
DDaria Antonenko, ${ }^{1}$ Florian Schubert, ${ }^{2}$ @Florian Bohm, ${ }^{1}$ Bernd Ittermann, ${ }^{2}$ Semiha Aydin, ${ }^{2}$ Dayana Hayek, ${ }^{1}$ \\ DUlrike Grittner, ${ }^{3,4}$ and Agnes Flöel ${ }^{1,3,5}$ \\ ${ }^{1}$ Department of Neurology, NeuroCure Clinical Research Center, Charité Universitätsmedizin, 10117 Berlin, Germany, ${ }^{2}$ Physikalisch-Technische \\ Bundesanstalt (PTB), 10587 Berlin, Germany, ${ }^{3}$ Center for Stroke Research and ${ }^{4}$ Department of Biostatistics and Clinical Epidemiology, Charité \\ Universitätsmedizin, 10117 Berlin, Germany, and ${ }^{5}$ Department of Neurology, University Medicine Greifswald, 17474 Greifswald, Germany
}

Transcranial direct current stimulation (tDCS) modulates human behavior, neuronal patterns, and metabolite concentrations, with exciting potential for neurorehabilitation. However, the understanding of tDCS-induced alterations on the neuronal level is incomplete, and conclusions from young adults, in whom the majority of studies have been conducted, cannot be easily transferred to older populations. Here, we investigated tDCS-induced effects in older adults $(N=48$; age range, $50-79$ years $)$ using magnetic resonance spectroscopy to quantify GABA levels as well as resting-state functional magnetic resonance imaging to assess sensorimotor network strength and interhemispheric connectivity. In a randomized, counterbalanced, crossover design, we applied anodal tDCS (atDCS), cathodal tDCS (ctDCS), and sham tDCS (stDCS) over the left sensorimotor region. We observed a significant reduction of GABA levels after atDCS compared with stDCS, reflecting the preserved neuromodulatory effect of atDCS in older adults. Moreover, resting-state functional coupling was decreased during atDCS compared with stDCS, most likely indicating augmented efficiency in brain network functioning. Increased levels of interhemispheric connectivity with age were diminished by atDCS, suggesting stimulation-induced functional decoupling. Further, the magnitude of atDCS-induced local plasticity was related to baseline functional network strength. Our findings provide novel insight into the neuronal correlates underlying tDCS-induced neuronal plasticity in older adults and thus might help to develop tDCS interventions tailored to the aging brain.

Key words: aging; magnetic resonance spectroscopy; plasticity; sensorimotor network; transcranial electrical stimulation

Significance Statement

Transcranial direct current stimulation (tDCS) modulates human behavior, neuronal patterns, and metabolite concentrations, with exciting potential for neurorehabilitation. However, the understanding of tDCS-induced alterations on the neuronal level is incomplete, and conclusions from young adults cannot be easily transferred to older populations. We used a systematic multimodal imaging approach to investigate the neurophysiological effects of tDCS in older adults and found stimulation-induced effects on GABA levels, reflecting augmented local plasticity and functional connectivity, suggesting modulation of network efficiency. Our findings may help to reconcile some of the recent reports on the variability of tDCS-induced effects, not only implicating age as a crucial modulating factor, but detailing its specific impact on the functionality of neural networks.

\section{Introduction}

Transcranial direct current stimulation (tDCS) has been shown to modulate human behavior as a result of stimulation-induced

Received Jan. 9, 2017; revised Feb. 17, 2017; accepted March 1, 2017.

Author contributions:D.A. and A.F. designed research;D.A., F.S., F.B., B.I., and S.A. performed research; D.A., F.S., B.I., S.A., D.H., and U.G. analyzed data; D.A. and A.F. wrote the paper.

This work was supported by the Bundesministerium für Bildung und Forschung (Grant 01G01424A) and the German-Israeli Foundation for Scientific Research and Development (Grant I-1299-105.4/2015). We thank two anonymous reviewers for their helpful comments on an earlier version of the manuscript.

The authors declare no competing financial interests.

Correspondence should be addressed to either of the following: Dr. Daria Antonenko, Department of Neurology, NeuroCure Clinical Research Center, Charité Universitätsmedizin, Charitéplatz 1, 10117 Berlin, Germany, neuronal changes in brain activity, connectivity, and metabolite concentrations (for review, see Nitsche et al., 2015; Fertonani and Miniussi, 2016). Effective modulation of behavioral performance through tDCS has been observed in various cognitive and motor domains, with the majority of work focusing on healthy young subjects (Nitsche et al., 2015; Perceval et al., 2016). Although findings of tDCS-induced behavioral improvement are promising, its efficacy has been challenged (Horvath et al., 2015; Minarik

E-mail: daria.antonenko@charite.de; or Dr. Agnes Flöel, Department of Neurology, University Medicine Greifswald, Ferdinand-Sauerbruch-Straße, 17474 Greifswald, Germany, E-mail: agnes.floeel@uni-greifswald.de.

DOI:10.1523/JNEUROSC1.0079-17.2017

Copyright $\odot 2017$ the authors $\quad 0270-6474 / 17 / 374065-09 \$ 15.00 / 0$ 
et al., 2016), stressing the need to scrutinize the neurophysiological effects of tDCS in specific conditions like aging and neurological diseases (Venkatakrishnan and Sandrini, 2012).

The concentration of brain metabolites such as GABA in the human brain can be assessed with magnetic resonance spectroscopy (MRS; Stagg et al., 2011; Puts and Edden, 2012; Mullins et al., 2014). GABA as the principle inhibitory neurotransmitter is associated with a variety of cognitive and neuronal functions (Puts and Edden, 2012). In young adults, anodal tDCS (atDCS) has been found to induce a reduction of GABA levels within primary sensorimotor cortices (SM1; Stagg et al., 2009a; Kim et al., 2014; Bachtiar et al., 2015), while cathodal tDCS (ctDCS) led to a reduction of glutamate $(\mathrm{Glu})$ levels with a correlated decrease of GABA (Stagg et al., 2009a) or had no effect on MRS-assessed metabolites (Kim et al., 2014). A local decrease of GABA levels in SM1 is one of the prerequisites for synaptic plasticity, thus representing an important determinant of human motor learning (Stagg and Nitsche, 2011; Stagg et al., 2011; Stagg, 2014). Further, the degree of GABA responsiveness to atDCS correlated positively with learning and memory in the motor domain (Kim et al., 2014). However, other studies have failed to detect atDCS effects on GABA levels (Tremblay et al., 2016; Wilke et al., 2017).

Intrascanner tDCS allows concomitant assessment of functional magnetic resonance imaging (fMRI; for pioneer work, see Antal et al., 2011). Here, resting-state fMRI (rs-fMRI) has become prominent in the probing of tDCS-induced functional connectivity (FC) alterations independent of task-related activity and performance (Fox and Raichle, 2007; Sehm et al., 2012). In young adults, widespread connectivity changes have been reported as a result of tDCS, with observations of both augmented coupling within networks of interest (Stagg et al., 2009b; Keeser et al. 2011; due to ctDCS, Amadi et al., 2014; due to atDCS, Bachtiar et al., 2015) and reduced interhemispheric FC (due to atDCS, Alon et al., 2011; Polanía et al., 2011a; Sehm et al., 2013). Two recent studies combining MRS and fMRI approaches have found an inverse association between sensorimotor network (SMN) strength and local inhibitory tone within SM1 in young adults (Stagg et al., 2014; Bachtiar et al., 2015).

Within the context of tDCS, not only as a tool in neuroscientific research but also as a potential interventional means to counteract age-related cognitive and neuronal deterioration like in dementia and stroke, the study of older adults is of high relevance (Crosson et al., 2015; Perceval et al., 2016). Because of substantial age-related alterations in the older human brain with regard to structure, connectivity, neurotransmitter levels, and ability to induce long-term potentiation (Hedden and Gabrieli, 2004; Burke and Barnes, 2006; Bishop et al., 2010; Gutchess, 2014; Sala-Llonch et al., 2015), stimulation effects on the neuronal level may differ substantially from those in young healthy brains (Meinzer et al., 2012, 2013; Perceval et al., 2016). Thus, elucidating the neuronal network patterns induced by tDCS in older adults may help to pave the way toward the development of stimulation protocols tailored specifically to the aging brain.

Here, we systematically investigated tDCS-induced effects on the local and network level in older adults using MRS to assess GABA levels and rs-fMRI to assess FC. We chose a well established electrode montage, which has been reliably shown in young adults to modulate motor learning (Nitsche et al., 2003), as well as FC within the SMN and local inhibitory tone of SM1 (Stagg and Nitsche, 2011; Sehm et al., 2013; Bachtiar et al., 2015).
Table 1. Participant characteristics

\begin{tabular}{lccc}
\hline & $\begin{array}{c}\text { Whole group } \\
(N=48)\end{array}$ & $\begin{array}{c}\text { Y0 group } \\
(N=24)\end{array}$ & $\begin{array}{c}\text { 00 group } \\
(N=24)\end{array}$ \\
\hline Age, years & $63.3(8.0)$ & $56.6(4.4)$ & $70.0(4.1)^{*}$ \\
Education, years & $15.1(2.5)$ & $15.3(2.5)$ & $14.9(2.5)$ \\
Handedness $^{\circ}$ & $90.2(24.4)$ & $86.7(32.8)$ & $93.8(10.6)$ \\
Semantic fluency, $N$ & $23.6(5.6)$ & $23.7(6.2)$ & $23.5(5.2)$ \\
Boston Naming Test, $N$ (maximum 15) & $14.7(0.5)$ & $14.8(0.4)$ & $14.6(0.5)$ \\
Mini-Mental State (maximum 30) & $29.3(0.9)$ & $29.4(0.8)$ & $29.2(0.9)$ \\
Word list learning, $N$ & & & \\
$\quad$ Total & $22.4(3.4)$ & $22.9(2.7)$ & $21.9(3.9)$ \\
$\quad$ Trial 1 & $5.9(1.5)$ & $6.1(1.3)$ & $5.8(1.7)$ \\
$\quad$ Trial 2 & $7.7(1.3)$ & $7.8(1.1)$ & $7.5(1.4)$ \\
$\quad$ Trial 3 & $8.8(1.1)$ & $9.0(0.8)$ & $8.6(1.3)$ \\
Word list retrieval, $N$ & $8.2(1.3)$ & $8.5(1.1)$ & $7.9(1.4)$ \\
Word list intrusions, $N$ & $0.9(1.6)$ & $0.9(1.9)$ & $0.8(1.4)$ \\
Figure copying, $N$ (maximum 11) & $10.9(0.5)$ & $10.9(0.4)$ & $10.8(0.6)$ \\
Figure retrieval, $N$ (maximum 11) & $10.4(1.2)$ & $10.6(1.2)$ & $10.3(1.2)$ \\
Phonemic fluency, $N$ & $15.3(4.2)$ & $15.0(4.1)$ & $15.7(4.2)$ \\
Trail-making test time, S & & & \\
$\quad$ Part A & & & \\
Part B & $40.4(11.6)$ & $36.8(9.4)$ & $44.0(12.7)^{*}$ \\
\hline
\end{tabular}

Data are shown as the mean (SD). 00, old-old; $Y 0$, young-old.

${ }^{a}$ According to the Edinburgh Handedness Inventory.

${ }^{*} p<0.05$ for age group comparison.

\section{Materials and Methods}

Participants and study design

Forty-eight healthy older adults [24 female; mean (SD) age/age range, 63 years ( 8 years)/50-79 years] participated in the study. All were native German speakers, had no history of neurological or psychiatric disorders, and did not take CNS-acting medication. Smoking was not an exclusion criterion, but the proportion of smokers in the study sample was low (with 5 active smokers among 48 participants). Participants underwent neuropsychological testing before study inclusion to assure normal cognitive functioning [CERAD-Plus Test Battery, Consortium to Establish a Registry for Alzheimer's Disease, (https://www.memoryclinic.ch)]. Performance levels on all cognitive domains lay within age- and educationrelated norms. Participant characteristics, including demographic and cognitive testing performance data, are presented in Table 1 . The study was approved by the Ethics Committee of the Charite Universitätsmedizin and conducted in accordance with the Declaration of Helsinki. Written informed consent was obtained from all participants before participation.

All older adults participated in three sessions where atDCS, ctDCS, or sham tDCS (stDCS) was applied. Participants, the investigators performing the MRS/fMRI experiments and MRS data analyses, were blinded to the stimulation condition. The order of stimulation conditions was counterbalanced across subjects, and sessions were separated by at least 1 week. In each session, MRS measurement was acquired before and after tDCS, and resting-state fMRI was assessed during the 15-min tDCS application (Fig. 1A).

\section{$t D C S$}

Direct current stimulation was delivered through a battery-driven MRIcompatible stimulator (neuroConn DC-Stimulator Plus, neuroCare Group) positioned outside the scanner room. Two filter boxes, absorbing radio frequency noise, were placed between stimulator and electrodes inside and outside the scanner and $5 \mathrm{k} \Omega$ resistors were included in each electrode cable. This setting has been described previously in detail (Lindenberg et al., 2013; Meinzer et al., 2014). The "active" electrode (anode during atDCS and cathode during ctDCS) was inserted into a $5 \times 7 \mathrm{~cm}^{2}$ saline-soaked synthetic sponge and centered over the left SM1 (Fig. 1B). The reference electrode $\left(10 \times 10 \mathrm{~cm}^{2}\right)$ was positioned over the contralateral (right) supraorbital region. Electrode positions were individually determined according to the 10-20 EEG system (active electrode centered over C3). Electrode placements were verified both on the localizer scan in the beginning of MRI assessment and on each individual T1- 


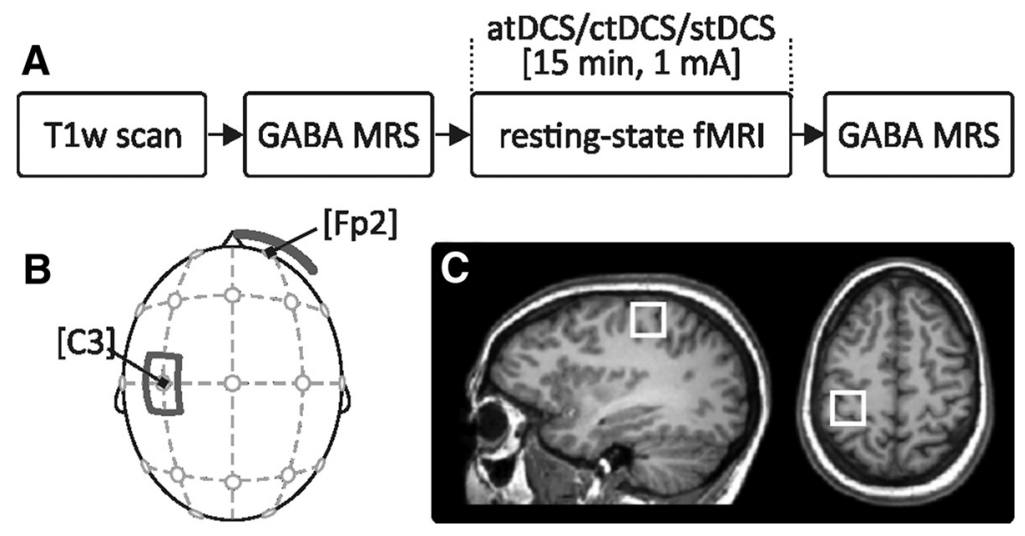

Figure 1. Illustration of experimental design, electrode montage for tDCS, and voxel positioning for MRS. $A$, Experimental design; all subjects underwent atDCS, ctDCS, and stDCS sessions separated by at least 1 week; the order of stimulation conditions was counterbalanced. GABA was measured immediately before and after $\mathrm{TDCS}$, and resting-state fMRI was assessed during tDCS. B, tDCS montage; active electrode over the left sensorimotor cortex (according to the 10-20 EEG system; (3), reference electrode over the right supraorbital area (sizes, $5 \times 7$ and $10 \times 10 \mathrm{~cm}^{2}$ ). C, MRS voxel positioning on an individual T1-weighted scan over the left sensorimotor area. Voxel size: $22 \times 22 \times 22 \mathrm{~mm}^{3}$. T1w, T1-weighted scan.

weighted image after scanning (described below). Resistance was monitored continuously during the stimulation interval. In the atDCS and ctDCS conditions, stimulation was delivered continuously for $15 \mathrm{~min}$ (with $10 \mathrm{~s}$ fade in/out intervals) with a constant current of $1 \mathrm{~mA}$, covering both fMRI sequences (described below). In the stDCS condition, stimulation was turned off after $30 \mathrm{~s}$.

Before and after each stimulation condition, mood ratings were administered using the positive affect (PA) and negative affect (NA) schedule (Watson et al., 1988). Participants rated their positive and negative affect ( 10 items each) on a scale ranging from 1 to 5 , where higher values describe more positive or negative feelings, respectively. After completion of the third experimental session, participants were asked to retrospectively report the occurrence of adverse effects (pain, tingling, itching, burning, fatigue, tension, headache, and discomfort) during stimulation in a standardized questionnaire (which was adapted from Poreisz et al., 2007).

\section{MRI acquisition}

MR images and spectra were acquired on a 3T Verio scanner (Siemens Healthcare), using a 32-channel receive-only head coil. After the recording of a scout image, high-resolution anatomical images were acquired using a three-dimensional T1-weighted magnetization-prepared rapid acquisition gradient echo [repetition time (TR), $2300 \mathrm{~ms}$; echo time (TE), $3.03 \mathrm{~ms}$; inversion time, $900 \mathrm{~ms}$; flip angle, $9^{\circ}$; $256 \times 256 \times 192$ matrix; $1 \mathrm{~mm}^{3}$ isotropic voxel). This image was used to place the spectroscopic voxel of interest $\left(22 \times 22 \times 22 \mathrm{~mm}^{3}\right)$ over the left precentral hand knob (Yousry et al., 1997; Fig. 1C). For MRS, first the transmitter radiofrequency voltage was calibrated for the individual volume of interest, followed by the adjustment of all first- and second-order shims using FAST(EST)MAP (Gruetter, 1993; Gruetter and Tkác, 2000). GABA-edited spectra were recorded using the MEGA-PRESS technique (Mescher et al., 1998; Edden et al., 2012). The number of acquired scans was 256 using a TR of $3000 \mathrm{~ms}$ and a TE of $68 \mathrm{~ms}$. Immediately afterward, a spectrum without water suppression was recorded (eight acquired scans). Due to its editing scheme, MEGA-PRESS permits the discernment of the GABA pseudo-triplet at $3.0 \mathrm{ppm}$ with excellent selectivity and good sensitivity. The editing pulse was applied to the C3-GABA resonance at $1.9 \mathrm{ppm}$ (edited scan), while in the alternate scan the editing pulse was applied at $1.5 \mathrm{ppm}$ (unedited scan). The acquisition of restingstate $\mathrm{fMRI}$ was performed using two consecutive echoplanar imaging (EPI) sequences $\left(3 \times 3 \times 4 \mathrm{~mm}^{3}\right.$; TR, $2300 \mathrm{~ms}$; TE, $30 \mathrm{~ms}$; flip angle, $90^{\circ}$; 35 slices; no gap; interleaved acquisition; field of view, $192 \times 192 \mathrm{~mm}^{2}$; matrix, $64 \times 64 ; 150$ volumes). Subjects were instructed to keep their eyes closed, to try not to fall asleep, and to think of nothing particular. None of the participants had fallen asleep during the stimulation interval, as evinced from oral interviews after scanning.

\begin{abstract}
MRI analysis
Structural analyses. To quantify individual brain atrophy, we used the T1-weighted images to estimate individual brain tissue volume, normalized for subject head size, with SIENAX (Smith et al., 2002), part of the FMRIB Software library (FSL; Smith et al., 2004; Jenkinson et al., 2012). To assess the impact of cortical atrophy, brain gray matter volume was included as an additional covariate in statistical analyses (described below). In addition, the localization of MRS voxel positions in the sensorimotor cortex was verified using the Harvard-Oxford anatomical atlas as implemented in FSL after linear registration of the center coordinates into MNI space (Jenkinson and Smith, 2001).
\end{abstract}

MRS. Edited spectra were analyzed using LCModel (Provencher, 1993) with a measured basis set containing GABA, $N$-acetylaspartate (NAA), Glu, glutamine, and glutathione and referenced to the concentrations used for creating the basis set. For fitting of the unedited spectrum, a simulated basis set containing 16 metabolites was used (i.e., alanine, aspartate, phosphocholine, creatine, phosphocreatine, GABA, Glu, glutamine, myoinositol, lactate, NAA, scyllo-inositol, taurine, glucose, $N$-acetylaspartylglutamate, and glycerophosphocholine). Only those GABA amplitudes returned by LCModel for which the Cramér-Rao lower bounds (CRLBs) were $<35 \%$ were considered for further analysis [mean (SD) CRLB, 17.3 (5.1); note, however that CRLBs of only two participants were $>30 \%$; Bhattacharyya et al., 2011; Brady et al., 2013; Tremblay et al., 2016]. CRLBs for Glu were $<12 \%$. GABA and Glu concentrations were quantified as the ratio to total creatine ( $\mathrm{tCr}$ ) fitted from the unedited spectra (Mullins et al., 2014; Stagg, 2014).

rs-fMRI. Image analysis was performed using tools from FSL (http:// fsl.fmrib.ox.ac.uk/fsl/fslwiki/; RRID:SCR_002823; Jenkinson et al., 2012). Preprocessing of individual 4 D datasets included motion correction, brain extraction, spatial smoothing using a Gaussian kernel of fullwidth half-maximum of $6 \mathrm{~mm}$, and high-pass temporal filtering of $150 \mathrm{~s}$ $(0.007 \mathrm{~Hz})$. Functional images were aligned to individual structural T1weighted images using boundary-based registration (Greve and Fischl, 2009) and then to standard space (MNI, $2 \mathrm{~mm}$ ) using the nonlinear registration tool. All 4D datasets were visually scanned for severe artifacts, and all images were examined after each processing step. Datasets of individuals with severe artifacts that were not removed after preprocessing were excluded from further analyses.

For seed-based analyses, center coordinates of the MRS voxel were surrounded by a sphere of $8 \mathrm{~mm}$ radius to obtain individual regions of interest (ROIs) for each experimental session. Coordinates from the three conditions per subject were averaged to obtain a single individual SM1 seed per subject, registered to MNI space. Left ROIs were flipped around the midline, resulting in individual right SM1 ROIs (Stagg et al., 2014). The time course of the resting-state BOLD signal was extracted from each subject's left SM1 seed images for each stimulation condition. The resulting time courses were then correlated between hemispheres to give a measure of interhemispheric FC. An $8 \mathrm{~mm}$ sphere around the center of the left dorsal premotor cortex (PMd) based on the connectivity-based parcellation atlases implemented in FSL (Tomassini et al., 2007) was created as a control ROI. As Pearson's $r$ is not normally distributed, the resulting $r$ values were transformed into $z$ scores using Fisher $r$-to- $z$ transformation. As head motion may confound with ROIbased connectivity and bias between-group comparisons most probably in the direction of the assumed difference (Van Dijk et al., 2012), we calculated head movement from the translation parameters from the rigid body correction of head motion (Jenkinson et al., 2002). Mean head motion parameters that were extracted from preprocessing represent the mean displacement in millimeters of each brain volume compared with the previous volume (Van Dijk et al., 2012). 
A

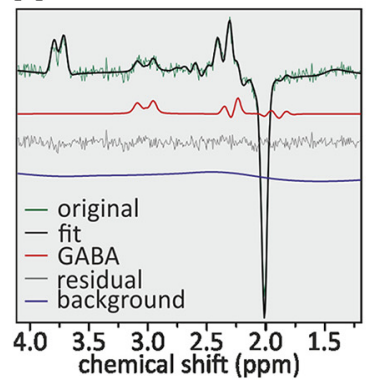

B

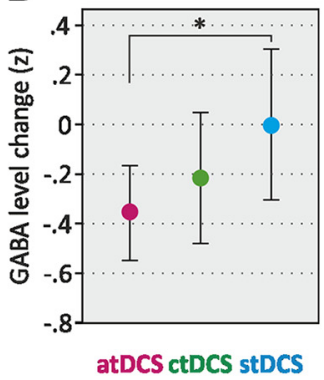

C

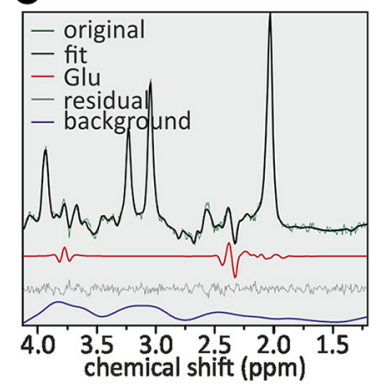

D

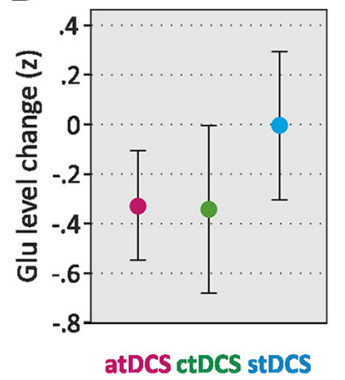

Figure 2. Results from MRS acquisition for GABA and Glu levels. $\boldsymbol{A}, \boldsymbol{C}$, Sample magnetic resonance spectra from the sensorimotor voxel. MEGA-PRESS difference spectrum ( $\boldsymbol{A}$ ) and MEGA-PRESS unedited spectrum (C). Top lines of each figure box represent the original spectrum and the fit, respectively. Also shown are fits for $G A B A$ and $G l u$, as well as the background. The small residuals reflect the high quality of the fits. $\boldsymbol{B}$, Change of GABA levels by condition. GABA levels after atDCS were significantly reduced compared with stDCS. $D$, Change of Glu levels by condition. GABA and Glu level changes as ratios to $\mathrm{t} C \mathrm{r}$ normalized to sham condition ( $z$-values). Error bars represent the $95 \% \mathrm{Cl}$. ${ }^{*} p \leq 0.05$.

For SMN analysis, independent component analyses (ICAs) were performed using the MELODIC (Multivariate Exploratory Linear Optimized Decomposition into Independent Components) tool 3.14 from FSL (Beckmann et al., 2005). All individual data were temporally concatenated across subjects and conditions to create a single $4 \mathrm{D}$ dataset. This dataset was then decomposed into 20 independent components. Components of interest [i.e., the sensorimotor and default mode network (DMN) used as control ROI] were selected by visual inspection based on previous literature (Beckmann et al., 2005). Next, the dual-regression approach was used to identify separate maps for each subject and each condition. This involved a spatial regression of these components against each individual $4 \mathrm{D}$ dataset and, subsequently, a temporal regression of the resulting time series to generate subject- and condition-specific spatial maps. Those maps reflect the subject- and condition-specific strength of FC within the respective network. The resulting network $z$-maps were then masked by the group mean map of the respective component, and the mean values extracted for each subject and condition were used to quantify the strength of FC within the network (Binnewijzend et al., 2012; Bachtiar et al., 2015). Both seed-based and ICA-based FC analysis approaches closely resemble the analyses by Amadi et al. (2014), Stagg et al. (2014), and Bachtiar et al. (2015).

\section{Statistical analysis}

SPSS Statistics 23 [IBM; RRID:SCR_002865 (http://www-01.ibm.com/ software/uk/analytics/spss/)] was used for statistical analyses. For condition effects, linear mixed models (random intercept models; Verbeke and Molenberghs, 2000) were calculated for each dependent variable with repeated measurements as level-one units nested in individuals who were level-two units. Models included the factor condition (atDCS, ctDCS, stDCS), the variable age, and their interaction, where appropriate. Model-based post hoc pairwise comparisons of the estimated fixed effects were computed. The difference in adverse effects between conditions was analyzed with logistic mixed-model analysis adjusted for age and sex. Pearson's correlation coefficients were computed for linear associations between variables. A two-sided significance level of $\alpha=0.05$ was used.

\section{Results}

\section{tDCS-induced effects on GABA levels}

A sample magnetic resonance spectrum for GABA is depicted in Figure $2 A$. Linear mixed-model analysis $(N=47$ participants/ 134 data points) revealed a significant difference in GABA levels between conditions $\left(F_{(2,87)}=3.61, p=0.031\right.$; Fig. $2 B$, Table 2$)$. Post hoc comparisons of model-based means showed that the GABA level was reduced in atDCS compared with stDCS [mean difference, -0.14 (95\% CI, -0.26 to 0.02$), p=0.022$ ]. This effect was polarity specific as there was no significant difference between ctDCS and stDCS [mean difference, -0.09 (95\% CI, -0.21 to 0.04$), p=0.165]$. The effect of age was not significant $\left(F_{(1,46)}=0.39, p=0.537\right)$, but there was an interaction between condition and age $\left(F_{(2,87)}=4.23, p=0.018\right)$. To explore the
Table 2. Change (\%) in GABA and Glu levels for the sensorimotor voxel after stimulation

\begin{tabular}{lllc}
\hline & atDCS & ctDCS & stDCS \\
\hline GABA change & $7.6(25.2)$ & $11.7(34.7)$ & $18.4(37.9)$ \\
Glu change & $0.7(6.5)$ & $0.0(9.6)$ & $3.4(8.7)$ \\
\hline
\end{tabular}

Data are shown as mean (SD).

meaning of this interaction, we divided the age group using a median split [median, 63 years; young-old ( $\mathrm{YO}),<63$ years; oldold $(\mathrm{OO}),>63$ years]. This distinction revealed that the atDCS effect was larger in the older age group [i.e., in the OO adults $\left(>63\right.$ years; $\left.F_{(2,87)}=5.54, p=0.005\right]$ with mean GABA reduction in atDCS versus stDCS of -0.23 ( $95 \% \mathrm{CI},-0.40$ to 0.06$)$ compared with $\mathrm{YO}$ with -0.04 ( $95 \% \mathrm{CI},-0.21$ to 0.13$)$. A sample magnetic resonance spectrum for Glu is depicted in Figure 2C. The comparison of Glu changes did not show a statistically significant effect of condition $\left(F_{(2,89)}=0.39, p=0.69\right)$ or age $\left(F_{(1,48)}=1.69, p=0.20\right.$; Fig. $2 D$, Table 2$)$. However, exploratory post hoc comparisons revealed lower levels of Glu after ctDCS compared with stDCS [mean difference, -0.03 (95\% CI, -0.06 to 0.001$), p=0.057$ ] and after atDCS compared with stDCS [mean difference, -0.03 ( $95 \% \mathrm{CI},-0.06$ to 0.003$), p=0.076$ ].

\section{tDCS-induced effects on interhemispheric FC and SMN}

Sample positions of left and right ROIs in the sensorimotor cortices are illustrated in Figure $3 A$. The interhemispheric FC differed between conditions (linear mixed models, $N=44$ participants/129 data points; $\left.F_{(2,84)}=3.73, p=0.028\right)$. Post hoc comparisons demonstrated a lower interhemispheric FC in atDCS compared with stDCS [mean difference - 0.06 (95\% CI, -0.11 to 0.01$), p=0.033$ ] and ctDCS [mean difference, -0.07 (95\% CI, 0.01 to 0.12 ), $p=0.013$; Fig. $3 B$ ]. Further, higher age was associated with higher interhemispheric FC [mean effect per decade, 0.05 ( $95 \%$ CI, 0.01 to 0.11 ); i.e., FC is elevated by 0.05 for each decade of life $\left.\left(F_{(1,43)}=4.20, p=0.047\right)\right]$. There was no significant difference between conditions for FC between left SM1 and PMd $\left(F_{(2,84)}=1.11, p=0.31\right)$. Head motion did not differ between conditions [mean: atDCS, 0.16 (95\% CI, 0.14 to 0.19); ctDCS, 0.15 (95\% CI, 0.13 to 0.18 ); stDCS, 0.17 (95\% CI, 0.14 to 0.19$) ; F_{(2,84)}=0.58, p=0.564$ ] but did differ between age groups in the direction of higher motion with higher age [mean: YO, 0.13 (95\% CI, 0.10 to 0.17 ); OO, 0.19 (95\% CI, 0.16 to 0.22 ); $\left.F_{(1,42)}=5.14, p=0.029\right]$. Including head motion as an additional covariate in the statistical model did not show a significant effect on interhemispheric $\mathrm{FC}\left(F_{(1,114)}=1.28, p=0.260\right)$. 
A

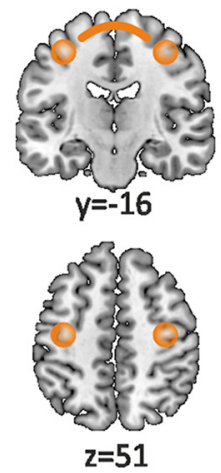

B

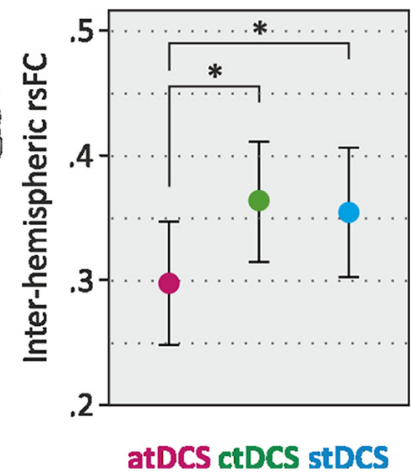

C

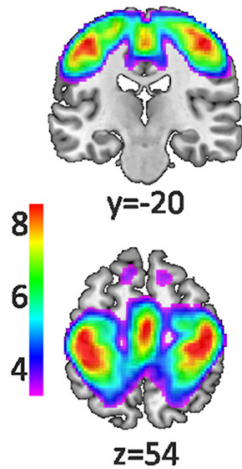

D

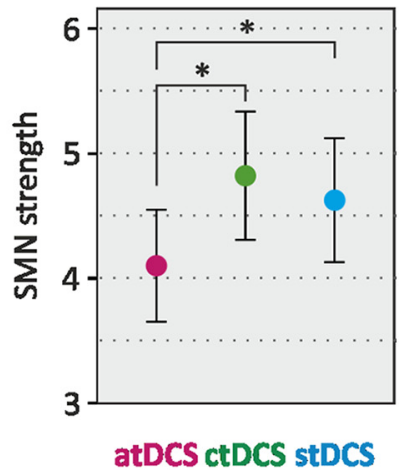

Figure 3. Results from rs-fMRI acquisition for interhemispheric functional connectivity and sensorimotor network strength. $\boldsymbol{A}$, Illustration of left and right ROls in the sensorimotor cortex. ROls were derived individually from MRS voxel center coordinates (flipped around the midline for right ROI). $\boldsymbol{B}$, Interhemispheric connectivity by condition (Fisher $r$ to $z$ values). $\boldsymbol{C}$, Sensorimotor network as derived from ICA analysis. D, Strength in the sensorimotor network by condition (arbitrary units). Error bars represent the $95 \% \mathrm{Cl}$. ${ }^{*} p \leq 0.05$.

SMN as derived from the ICA is illustrated in Figure 3C. SMN strength differed between conditions (linear mixed models, $N=$ 44 participants/129 data points; $\left.F_{(2,84)}=4.76, p=0.011\right)$. Post hoc comparisons revealed reduced SMN strength in atDCS compared with stDCS [mean difference, -0.52 (95\% CI, -1.00 to $0.03), p=0.039$ ] and ctDCS [mean difference, -0.74 (95\% CI, -1.22 to 0.25$), p=0.003$; Fig. $3 D$ ]. There was no significant effect of age $\left(F_{(1,42)}<0.01, p=0.96\right)$. DMN strength showed a negative association with age $\left[F_{(1,42)}=5.58, p=0.023\right.$; mean effect per decade, -0.53 ( $95 \%$ CI, -0.98 to 0.08$)$; i.e., DMN strength is reduced by -0.53 for each decade of life]. DMN

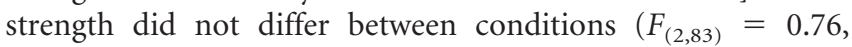
$p=0.47)$.

\section{Relationship between baseline GABA levels and SMN}

We observed an inverse correlation between GABA levels and overall strength within the SMN at baseline $\left(r_{(37)}=-0.31, p=\right.$ $0.067)$. Further explorative illustrations revealed that this association was evident only in the YO group $\left[(<63\right.$ years $) r_{(18)}=$ $-0.574, p=0.013$; OO group $(>63) r_{(19)}=0.097, p=0.692$; as defined by a median split, see above; Fig. $4 A$ ]. Note that for the YO group sensitivity analysis without the outlier yielded a similar Pearson's correlation coefficient $\left(r_{(17)}=-0.468, p=0.058\right)$.
A

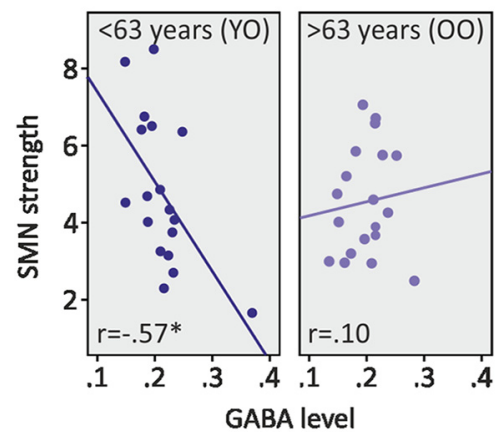

B

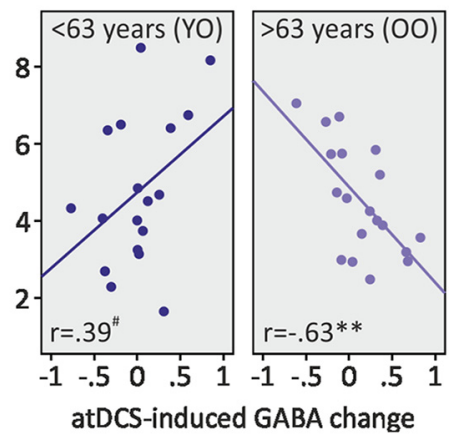

Figure 4. Relationship between GABA and sensorimotor network strength. $\boldsymbol{A}$, Association between baseline GABA levels and baseline SMN strength, separately for $Y 0$ and 00 adults (median split by 63 years of age). Note that for the $Y 0$ group sensitivity analysis without the outlier yielded a similar Pearson's correlation coefficient $\left(r_{(17)}=-0.468, p=0.058\right)$. $\boldsymbol{B}$, Association between $\mathrm{tDCS}$-induced change of local GABA levels (stDCS minus atDCS; larger numbers represent a higher disinhibitory effect produced by atDCS) and baseline SMN strength, separately for $\mathrm{Y} 0$ and 00 adults. GABA level was given as a ratio to $\mathrm{tCr}$. ${ }^{*} p \leq 0.05$, ${ }^{* *} p \leq 0.01, \# p \leq 0.10$.

\section{Associations of tDCS-induced effects with GABA levels and SMN}

We did not find a significant relationship between tDCS-induced effects on GABA levels and SMN strength $\left(r_{(37)}=0.21, p=\right.$ $0.215)$. Further exploratory analyses of associations between baseline SMN strength and tDCS-induced alterations of GABA levels revealed opposite correlations in the age groups $(<63$ and $>63$ years), while in the group $<63$ years there was a trend toward a positive association between tDCS effects on GABA with baseline SMN coupling $\left(r_{(19)}=0.389, p=0.096\right)$; in the older age group ( $>63$ years), we observed an inverse correlation $\left(r_{(18)}=\right.$ $-0.634, p=0.004$; Fig. $4 B)$.

\section{Resistance monitoring, mood ratings, and adverse effect questionnaire}

The mean (SD) resistance across all sessions at the beginning of stimulation was $16.28 \mathrm{k} \Omega(0.92 \mathrm{k} \Omega$; range, $13-18 \mathrm{k} \Omega)$ with no difference between conditions [atDCS, $16.36 \mathrm{k} \Omega(0.13 \mathrm{k} \Omega)$; ctDCS, $16.27 \mathrm{k} \Omega(0.12 \mathrm{k} \Omega)$; stDCS, $16.16 \mathrm{k} \Omega(0.17 \mathrm{k} \Omega) ; F_{(2,84)}=$ $0.52, p=0.60]$ and stayed stable across the stimulation interval with only small changes in $29 \%$ of experimental sessions. Note that contact medium of the electrodes may be particularly relevant in the concurrent acquisition of tDCS and MRI due to drying of saline-soaked electrode sponges during long acquisition times (Woods et al., 2016). Here, we did not observe a rise in resistance for the majority of our experimental sessions (i.e., 71\%) using electrode sponges. However, it may be advisable to use conductance paste in future tDCS-MRI experiments to avoid the prolongation of preparation times and to reduce the probability of resistance rise leading to pain and burning sensations, and to a termination of the stimulation.

PA and NA before and after the scanning session are displayed in Table 3. Neither positive nor negative affect ratings were significantly different between conditions $(N=48$ participants $/ 288$ data points; PA: $F_{(2,235)}=1.33, p=0.27$; NA: $\left.F_{(2,235)}=0.05, p=0.95\right)$. Time effects $\left(\mathrm{PA}: F_{(1,235)}=40.88, p<0.01 ; \mathrm{NA}\right.$ : 
Table 3. Participants' mood ratings before and after the scanning sessions

\begin{tabular}{llll}
\hline & atDCS & ctDCS & stDCS \\
\hline $\begin{array}{l}\text { Positive affect } \\
\quad \text { Before }\end{array}$ & $3.3(0.7)$ & $3.2(0.7)$ & $3.2(0.8)$ \\
$\quad$ After & $3.0(0.9)$ & $3.0(0.9)$ & $2.9(0.9)$ \\
$\begin{array}{l}\text { Negative affect } \\
\text { Before }\end{array}$ & & & \\
After & $1.1(0.2)$ & $1.2(0.3)$ & $1.1(0.2)$ \\
\hline
\end{tabular}

Data are shown as mean (SD).

Table 4. Adverse effects during stimulation

\begin{tabular}{lrrrl}
\hline & atDCS & ctDCS & stDCS & $p^{a}$ \\
\hline Pain & 5 & 8 & 6 & 0.139 \\
Tingling & 26 & 26 & 25 & 0.922 \\
Itchiness & 5 & 4 & 2 & 0.216 \\
Burning & 4 & 4 & 5 & 0.680 \\
Fatigue & 14 & 14 & 15 & 0.915 \\
Tension & 3 & 6 & 3 & 0.234 \\
Loss of concentration & 3 & 2 & 2 & 0.291 \\
Headache & 0 & 1 & 1 & 0.999 \\
Discomfort & 6 & 8 & 6 & 0.496 \\
\hline
\end{tabular}

Numbers correspond to $n$ participants (total $N=48$ ).

${ }^{a} p$ values are for comparison of conditions (adjusted for age and sex).

$\left.F_{(1,235)}=7.49 ; p<0.01\right)$ indicated reduced reporting of both positive and negative affect after stimulation [PA: mean difference, -0.28 (95\% CI, -0.35 to 0.19$), p<0.001$; NA: mean difference, -0.055 ( $95 \% \mathrm{CI},-0.10$ to 0.02$), p=0.007$ ]. Itchiness and fatigue were most commonly reported (by $56 \%$ and $33 \%$ of the participants). The occurrence of adverse effects did not differ between stimulation conditions (Table 4).

\section{Discussion}

This is the first study in older adults, spanning a wide age range (50-79 years), showing tDCS-induced plasticity in local inhibitory tone of the sensorimotor cortex in combination with restingstate FC. Local inhibitory tone was assessed using MRS to determine GABA levels in left SM1, a major node of the SMN and the target of the stimulation. Functional SMN connectivity was quantified by interhemispheric coupling between SM1 as well as by SMN strength. First, we observed an atDCS-induced reduction of GABA levels, reflecting neuroplastic alterations in brain chemistry. Second, resting-state functional coupling was decreased during atDCS compared with stDCS, most likely indicating augmented efficiency in brain network functioning. Third, while an association between higher baseline levels of functional coupling and lower GABA levels was observed, the magnitudes of tDCS-induced effects on these parameters were not correlated. Last, exploring an association of atDCS-induced GABA changes with baseline SMN strength revealed opposite directions in $\mathrm{YO}$ adults ( $<63$ years) and $\mathrm{OO}$ adults ( $>63$ years), possibly suggesting age-related differences in network integrity.

\section{atDCS reduced GABA levels in older adults}

Previous studies using transcranial magnetic stimulation to assess changes in GABAergic neurotransmission across the human life span have demonstrated a decline in cortical excitability and GABA-mediated intracortical inhibition within the motor system with advancing age (Heise et al., 2013; Bhandari et al., 2016). Preserved event-related modulation correlated with superior behavioral performance in older adults (Heise et al., 2013). In line with these observations, the "disinhibitory" effect of atDCS has been suggested to reflect a preserved modulatory capacity of the SMN in older adults (Heise et al., 2014; Bhandari et al., 2016). Whether or not GABAergic neurotransmission and plasticity within the SMN is in general negatively affected in the course of aging is still a matter of intense debate, as shown by a recent meta-analysis (Bhandari et al., 2016).

Our study is the first to use MRS to assess tDCS-induced alterations of GABA concentrations in older adults. We were able to demonstrate that atDCS over left SM1 compared with stDCS reduced GABA levels within the target region. The reduction was neurochemically specific as no effect was observed on Glu levels and was polarity specific as it was not observed after ctDCS. In young adults, atDCS has previously been shown to reduce GABA concentration in SM1 (Stagg et al., 2009a; Kim et al., 2014; Bachtiar et al., 2015). Note the elevation of GABA levels in stDCS, which is consistent with other tDCS studies that have likewise observed a temporal variability of MRS-assessed metabolites such as GABA (Kim et al., 2014) or glutamate (Hone-Blanchet et al., 2016) on a short time scale. The mechanisms underlying this increase are, so far, unknown but may be related to experimental instabilities (Harris et al., 2014) or the vigilance state of the participant, issues to be systematically addressed in future studies. Only two previous studies, both in young adults, have so far acquired MRS data after cathodal stimulation, with mixed results, as follows: while Kim et al. (2014) likewise did not find an effect after cathodal stimulation, Stagg et al. (2009a) reported reduced Glu levels after cathodal stimulation with a correlated decrease of GABA concentration, most likely due to the biochemical association between these neurotransmitters. To further explore the effect of ctDCS on Glu levels in our data, we computed exploratory post hoc tests that revealed a Glu level decrease compared with stDCS, thus supporting the results of Stagg et al. (2009a) also for older adults. However, the large interindividual variability in our group of older adults may have prevented the finding of statistical significant differences similar to those discussed by Tremblay et al. (2016).

In sum, our observation of reduced GABA levels after atDCS suggests the involvement of GABAergic neurotransmission in the neuronal effects of atDCS (targeting the SM1) in older adults, indicating preserved neuromodulatory tDCS effects on the sensorimotor system.

\section{atDCS decreased resting-state functional coupling in older adults}

Recently, the use of resting-state fMRI has become prominent in probing tDCS-induced FC modulations independent of taskrelated activity and performance, which may be especially advantageous in older adults (Fox and Raichle, 2007; Sehm et al., 2012). Here, we found an atDCS-induced reduction of interhemispheric FC in older adults, suggesting that atDCS targeting the SMN may act upon decoupling of the SM1 target and its contralateral homolog in this population. This finding partly corresponds to what has been shown in young adults (Alon et al., 2011; Polanía et al., 2011b; Sehm et al., 2013). In addition, in our sample of older adults, higher levels of interhemispheric coupling correlated with increased age. Age-related deterioration in structural connections that mediate the communication between hemispheres, resulting in reduced interhemispheric inhibition, have been well documented (for review, see Fling et al., 2011). The structural decline leads to an increase of interhemispheric functional synchronization with age, which has been related to age deficits in motor as well as cognitive performance (Langan et al., 2010; Fling et al., 2011; Antonenko et al., 2013). In this context, atDCS- 
induced interhemispheric decoupling in our sample of older adults may reflect the reversal of age-related FC increases (Lindenberg et al., 2013).

Previous evidence suggests increased coupling in the SMN after anodal stimulation (Stagg et al., 2014; Bachtiar et al., 2015). This tDCS-induced neuromodulatory effect was interpreted as efficiency enhancement within the targeted neuronal network in young adults. Contrary to these results, in our group of older adults, resting-state functional coupling within the SMN was reduced during atDCS compared with stDCS. These differences may be due to the complex functional network reorganization that occurs in the course of healthy aging, including age-related connectivity decreases (most prominently in the DMN) as well as increases within (e.g., dorsal anterior network) and between networks, suggesting alterations of the overall functional architecture (for review, see Sala-Llonch et al., 2015). Regarding SMN in aging, no uniform picture has so far emerged, with evidence for connectivity increases (Tomasi and Volkow, 2012; Song et al., 2014) as well as for no age-related changes (Geerligs et al., 2015). One tentative explanation for the findings in our sample of older adults would be that augmented coupling may reflect dysfunctional network "hyperconnectivity," which is reduced by atDCS, resulting in more distinct or efficient functional SMNs, respectively. These between-study differences, however, may be partially explained by differences in study design regarding the timing of resting-state fMRI, since tDCS-induced neurophysiological effects depend on whether stimulation is applied on-line (as in our study) or off-line (Nitsche et al., 2005; Stagg and Nitsche, 2011; Stagg et al., 2014; Bachtiar et al., 2015), which may also affect respective resting-state FC metrics.

In sum, in our sample of older adults, atDCS modulated functional coupling within the resting-state SMN. We found (1) decreased interhemispheric connectivity between left and right sensorimotor cortices as well as (2) decreased SMN coupling strength.

\section{Associations between GABA and functional coupling and their modulation with tDCS}

Our data showed an inverse relationship between GABA levels and network connectivity at baseline. This observation is consistent with previous reports in young adults (Kapogiannis et al., 2013; Stagg et al., 2014; Bachtiar et al., 2015). Together, these findings point toward a connection between local inhibitory tone within a major network node and functional network coupling in the direction that lower GABA levels are associated with higher network connectivity. Our further exploratory correlation analyses showed, however, that this relationship was evident only in $\mathrm{YO}$ adults ( $<63$ years of age). The absence of such an association in $\mathrm{OO}$ adults ( $>63$ years of age) may indicate disrupted neuronal functioning in older age. The magnitude of atDCS-induced alterations in GABA levels and SMN strength was not correlated, supporting the hypothesis that tDCS effects on GABAergic transmission and resting-state FC may be mediated by distinct underlying mechanisms or follow different time courses (Bachtiar et al., 2015).

A further important assumption is that sensorimotor plasticity (as quantified by tDCS-induced GABA changes) depends on the functional integrity of the underlying network (Heise et al., 2014). The authors speculated that preserved modulatory capacity in older adults may predict the disinhibitory effect of tDCS. In the context of aging, however, it is not clear yet how this preserved modulatory capacity, which reflects the functional integrity of the underlying network, is quantified (i.e., whether higher or lower coupling strength represents intact systems) due to the complexity of age-related functional network reorganization (Sala-Llonch et al., 2015). In our data, further exploratory analyses showed an interesting differential effect of atDCS-induced GABA responsiveness and network coupling in $\mathrm{YO}$ versus $\mathrm{OO}$ individuals: While in YO adults ( $<63$ years of age) higher atDCSinduced GABA reduction correlated with higher SMN (though not statistically significant), in $\mathrm{OO}$ adults ( $>63$ years of age) higher GABA reduction correlated with lower SMN. Tentatively, this opposite pattern may point toward differential functionality of the system, suggesting that whereas at a younger age the integrity of neuronal network implies higher coupling, in older age it is reflected by reduced hyperconnectivity (which may in turn predict preserved modulatory capacity).

\section{Summary and outlook}

Together, our findings add novel information about tDCSinduced neuronal effects in older adults using a counterbalanced, sham-controlled, multimodal imaging approach. Because of altered neuroplasticity and network dynamics with age, a detailed investigation of older adults covering a wide age range is paramount, not only to elucidate the complexity of tDCS effects on a neurophysiological level, but also to develop more individually tailored interventional protocols in health and disease.

Our results may help to reconcile some of the recent reports on the variability of tDCS-induced effects, not only identifying age as a modulating factor, but detailing its specific impact on the functionality of neural networks. Given that atDCS may help to reverse dysfunctional network hyperconnectivity, it will now be of key interest to assess whether repeated atDCS applications lead to sustained reversal of age-related dysfunctional networks and associated functional scores.

\section{References}

Alon G, Roys SR, Gullapalli RP, Greenspan JD (2011) Non-invasive electrical stimulation of the brain (ESB) modifies the resting-state network connectivity of the primary motor cortex: a proof of concept fMRI study. Brain Res 1403:37-44. CrossRef Medline

Amadi U, Ilie A, Johansen-Berg H, Stagg CJ (2014) Polarity-specific effects of motor transcranial direct current stimulation on fMRI resting state networks. Neuroimage 88:155-161. CrossRef Medline

Antal A, Polania R, Schmidt-Samoa C, Dechent P, Paulus W (2011) Transcranial direct current stimulation over the primary motor cortex during fMRI. Neuroimage 55:590-596. CrossRef Medline

Antonenko D, Brauer J, Meinzer M, Fengler A, Kerti L, Friederici AD, Flöel A (2013) Functional and structural syntax networks in aging. Neuroimage 83:513-523. CrossRef Medline

Bachtiar V, Near J, Johansen-Berg H, Stagg CJ (2015) Modulation of GABA and resting state functional connectivity by transcranial direct current stimulation. Elife 4:e08789. CrossRef Medline

Beckmann CF, DeLuca M, Devlin JT, Smith SM (2005) Investigations into resting-state connectivity using independent component analysis. Philos Trans R Soc Lond B Biol Sci 360:1001-1013. CrossRef Medline

Bhandari A, Radhu N, Farzan F, Mulsant BH, Rajji TK, Daskalakis ZJ, Blumberger DM (2016) A meta-analysis of the effects of aging on motor cortex neurophysiology assessed by transcranial magnetic stimulation. Clin Neurophysiol 127:2834-2845. CrossRef Medline

Bhattacharyya PK, Phillips MD, Stone LA, Lowe MJ (2011) In vivo magnetic resonance spectroscopy measurement of gray-matter and white-matter gamma-aminobutyric acid concentration in sensorimotor cortex using a motion-controlled MEGA point-resolved spectroscopy sequence. Magn Reson Imaging 29:374-379. CrossRef Medline

Binnewijzend MA, Schoonheim MM, Sanz-Arigita E, Wink AM, van der Flier WM, Tolboom N, Adriaanse SM, Damoiseaux JS, Scheltens P, van Berckel BN, Barkhof F (2012) Resting-state fMRI changes in Alzheimer's disease and mild cognitive impairment. Neurobiol Aging 33:2018-2028. CrossRef Medline 
Bishop NA, Lu T, Yankner BA (2010) Neural mechanisms of ageing and cognitive decline. Nature 464:529-535. CrossRef Medline

Brady RO Jr, McCarthy JM, Prescot AP, Jensen JE, Cooper AJ, Cohen BM, Renshaw PF, Ongür D (2013) Brain gamma-aminobutyric acid (GABA) abnormalities in bipolar disorder. Bipolar Disord 15:434-439. CrossRef Medline

Burke SN, Barnes CA (2006) Neural plasticity in the ageing brain. Nat Rev Neurosci 7:30-40. CrossRef Medline

Crosson B, McGregor KM, Nocera JR, Drucker JH, Tran SM, Butler AJ (2015) The relevance of aging-related changes in brain function to rehabilitation in aging-related disease. Front Hum Neurosci 9:307. CrossRef Medline

Edden RA, Intrapiromkul J, Zhu H, Cheng Y, Barker PB (2012) Measuring T2 in vivo with J-difference editing: application to GABA at 3 Tesla. J Magn Reson Imaging 35:229-234. CrossRef Medline

Fertonani A, Miniussi C (2016) Transcranial electrical stimulation: what we know and do not know about mechanisms. Neuroscientist. Advance online publication. Retrieved March 14, 2017. doi:10.1177/1073858416631966. CrossRef Medline

Fling BW, Peltier SJ, Bo J, Welsh RC, Seidler RD (2011) Age differences in interhemispheric interactions: callosal structure, physiological function, and behavior. Front Neurosci 5:38. CrossRef Medline

Fox MD, Raichle ME (2007) Spontaneous fluctuations in brain activity observed with functional magnetic resonance imaging. Nat Rev Neurosci 8:700-711. CrossRef Medline

Geerligs L, Renken RJ, Saliasi E, Maurits NM, Lorist MM (2015) A brainwide study of age-related changes in functional connectivity. Cereb Cortex 25:1987-1999. CrossRef Medline

Greve DN, Fischl B (2009) Accurate and robust brain image alignment using boundary-based registration. Neuroimage 48:63-72. CrossRef Medline

Gruetter R (1993) Automatic, localized in vivo adjustment of all first-and secondorder shim coils. Magn Reson Med 29:804-811. CrossRef Medline

Gruetter R, Tkác I (2000) Field mapping without reference scan using asymmetric echo-planar techniques. Magn Reson Med 43:319-323. CrossRef Medline

Gutchess A (2014) Plasticity of the aging brain: new directions in cognitive neuroscience. Science (New York) 346:579-582. CrossRef Medline

Harris AD, Glaubitz B, Near J, John Evans C, Puts NA, Schmidt-Wilcke T, Tegenthoff M, Barker PB, Edden RA (2014) Impact of frequency drift on gamma-aminobutyric acid-edited MR spectroscopy. Magn Reson Med 72:941-948. CrossRef Medline

Hedden T, Gabrieli JD (2004) Insights into the ageing mind: a view from cognitive neuroscience. Nat Rev Neurosci 5:87-96. CrossRef Medline

Heise KF, Zimerman M, Hoppe J, Gerloff C, Wegscheider K, Hummel FC (2013) The aging motor system as a model for plastic changes of GABAmediated intracortical inhibition and their behavioral relevance. J Neurosci 33:9039-9049. CrossRef Medline

Heise K-F, Niehoff M, Feldheim JF, Liuzzi G, Gerloff C, Hummel FC (2014) Differential behavioral and physiological effects of anodal transcranial direct current stimulation in healthy adults of younger and older age. Front Aging Neurosci 6:146. CrossRef Medline

Hone-Blanchet A, Edden RA, Fecteau S (2016) Online effects of transcranial direct current stimulation in real time on human prefrontal and striatal metabolites. Biol Psychiatry 80:432-438. CrossRef Medline

Horvath JC, Forte JD, Carter O (2015) Quantitative review finds no evidence of cognitive effects in healthy populations from single-session transcranial direct current stimulation (tDCS). Brain Stimul 8:535-550. CrossRef Medline

Jenkinson M, Smith S (2001) A global optimisation method for robust affine registration of brain images. Med Image Anal 5:143-156. CrossRef Medline

Jenkinson M, Bannister P, Brady M, Smith S (2002) Improved optimization for the robust and accurate linear registration and motion correction of brain images. Neuroimage 17:825-841. CrossRef Medline

Jenkinson M, Beckmann CF, Behrens TE, Woolrich MW, Smith SM (2012) FSL. Neuroimage 62:782-790. CrossRef Medline

Kapogiannis D, Reiter DA, Willette AA, Mattson MP (2013) Posteromedial cortex glutamate and GABA predict intrinsic functional connectivity of the default mode network. Neuroimage 64:112-119. CrossRef Medline

Keeser D, Meindl T, Bor J, Palm U, Pogarell O, Mulert C, Brunelin J, Möller HJ, Reiser M, Padberg F (2011) Prefrontal transcranial direct current stimulation changes connectivity of resting-state networks during fMRI. J Neurosci 31:15284-15293. CrossRef Medline

Kim S, Stephenson MC, Morris PG, Jackson SR (2014) tDCS-induced alterations in GABA concentration within primary motor cortex predict motor learning and motor memory: a $7 \mathrm{~T}$ magnetic resonance spectroscopy study. Neuroimage 99:237-243. CrossRef Medline

Langan J, Peltier SJ, Bo J, Fling BW, Welsh RC, Seidler RD (2010) Functional implications of age differences in motor system connectivity. Front Syst Neurosci 4:17. CrossRef Medline

Lindenberg R, Nachtigall L, Meinzer M, Sieg MM, Flöel A (2013) Differential effects of dual and unihemispheric motor cortex stimulation in older adults. J Neurosci 33:9176-9183. CrossRef Medline

Meinzer M, Antonenko D, Lindenberg R, Hetzer S, Ulm L, Avirame K, Flaisch T, Flöel A (2012) Electrical brain stimulation improves cognitive performance by modulating functional connectivity and task-specific activation. J Neurosci 32:1859-1866. CrossRef Medline

Meinzer M, Lindenberg R, Antonenko D, Flaisch T, Flöel A (2013) Anodal transcranial direct current stimulation temporarily reverses age-associated cognitive decline and functional brain activity changes. J Neurosci 33:12470-12478. CrossRef Medline

Meinzer M, Lindenberg R, Darkow R, Ulm L, Copland D, Floel A (2014) Transcranial direct current stimulation and simultaneous functional magnetic resonance imaging. J Vis Exp (86):e51730. CrossRef Medline

Mescher M, Merkle H, Kirsch J, Garwood M, Gruetter R (1998) Simultaneous in vivo spectral editing and water suppression. NMR Biomed 11:266272. Medline

Minarik T, Berger B, Althaus L, Bader V, Biebl B, Brotzeller F, Fusban T, Hegemann J, Jesteadt L, Kalweit L, Leitner M, Linke F, Nabielska N, Reiter T, Schmitt D, Spraetz A, Sauseng P (2016) The importance of sample size for reproducibility of tDCS effects. Front Hum Neurosci 10:453. CrossRef Medline

Mullins PG, McGonigle DJ, O’Gorman RL, Puts NA, Vidyasagar R, Evans CJ, Edden RA (2014) Current practice in the use of MEGA-PRESS spectroscopy for the detection of GABA. Neuroimage 86:43-52. CrossRef Medline

Nitsche MA, Schauenburg A, Lang N, Liebetanz D, Exner C, Paulus W, Tergau F (2003) Facilitation of implicit motor learning by weak transcranial direct current stimulation of the primary motor cortex in the human. J Cogn Neurosci 15:619-626. CrossRef Medline

Nitsche MA, Seeber A, Frommann K, Klein CC, Rochford C, Nitsche MS, Fricke K, Liebetanz D, Lang N, Antal A, Paulus W, Tergau F (2005) Modulating parameters of excitability during and after transcranial direct current stimulation of the human motor cortex. J Physiol 568:291-303. CrossRef Medline

Nitsche MA, Polania R, Kuo M-F (2015) Transcranial direct current stimulation: modulation of brain pathways and potential clinical applications. In Brain stimulation: methodologies and interventions (Reti IM, ed), pp. 233-254. Hoboken, NJ: Wiley Blackwell.

Perceval G, Flöel A, Meinzer M (2016) Can transcranial direct current stimulation counteract age-associated functional impairment? Neurosci Biobehav Rev 65:157-172. CrossRef Medline

Polanía R, Paulus W, Antal A, Nitsche MA (2011a) Introducing graph theory to track for neuroplastic alterations in the resting human brain: a transcranial direct current stimulation study. Neuroimage 54:2287-2296. CrossRef Medline

Polanía R, Nitsche MA, Paulus W (2011b) Modulating functional connectivity patterns and topological functional organization of the human brain with transcranial direct current stimulation. Hum Brain Mapp 32: 1236-1249. CrossRef Medline

Poreisz C, Boros K, Antal A, Paulus W (2007) Safety aspects of transcranial direct current stimulation concerning healthy subjects and patients. Brain Res Bull 72:208-214. CrossRef Medline

Provencher SW (1993) Estimation of metabolite concentrations from localized in vivo proton NMR spectra. Magn Reson Med 30:672-679. CrossRef Medline

Puts NA, Edden RA (2012) In vivo magnetic resonance spectroscopy of GABA: a methodological review. Prog Nucl Magn Reson Spectrosc 60: 29-41. CrossRef Medline

Sala-Llonch R, Bartrés-Faz D, Junqué C (2015) Reorganization of brain networks in aging: a review of functional connectivity studies. Front Psychol 6:663. CrossRef Medline

Sehm B, Schäfer A, Kipping J, Margulies D, Conde V, Taubert M, Villringer A, Ragert P (2012) Dynamic modulation of intrinsic functional connectiv- 
ity by transcranial direct current stimulation. J Neurophysiol 108:32533263. CrossRef Medline

Sehm B, Kipping J, Schäfer A, Villringer A, Ragert P (2013) A comparison between uni- and bilateral tDCS effects on functional connectivity of the human motor cortex. Front Hum Neurosci 7:183. CrossRef Medline

Smith SM, Zhang Y, Jenkinson M, Chen J, Matthews PM, Federico A, De Stefano N (2002) Accurate, robust, and automated longitudinal and cross-sectional brain change analysis. Neuroimage 17:479-489. CrossRef Medline

Smith SM, Jenkinson M, Woolrich MW, Beckmann CF, Behrens TE, Johansen-Berg H, Bannister PR, De Luca M, Drobnjak I, Flitney DE, Niazy RK, Saunders J, Vickers J, Zhang Y, De Stefano N, Brady JM, Matthews PM (2004) Advances in functional and structural MR image analysis and implementation as FSL. Neuroimage 23 [Suppl 1]:S208-S219. CrossRef Medline

Song J, Birn RM, Boly M, Meier TB, Nair VA, Meyerand ME, Prabhakaran V (2014) Age-related reorganizational changes in modularity and functional connectivity of human brain networks. Brain Connect 4:662-676. CrossRef Medline

Stagg CJ (2014) Magnetic resonance spectroscopy as a tool to study the role of GABA in motor-cortical plasticity. Neuroimage 86:19-27. CrossRef Medline

Stagg CJ, Nitsche MA (2011) Physiological basis of transcranial direct current stimulation. Neuroscientist 17:37-53. CrossRef Medline

Stagg CJ, Best JG, Stephenson MC, O’Shea J, Wylezinska M, Kincses ZT, Morris PG, Matthews PM, Johansen-Berg H (2009a) Polarity-sensitive modulation of cortical neurotransmitters by transcranial stimulation. J Neurosci 29:5202-5206. CrossRef Medline

Stagg CJ, O'Shea J, Kincses ZT, Woolrich M, Matthews PM, Johansen-Berg H (2009b) Modulation of movement-associated cortical activation by transcranial direct current stimulation. Eur J Neurosci 30:1412-1423. CrossRef Medline

Stagg CJ, Bachtiar V, Johansen-Berg H (2011) What are we measuring with GABA magnetic resonance spectroscopy? Commun Integr Biol 4:573575. CrossRef Medline

Stagg CJ, Bachtiar V, Amadi U, Gudberg CA, Ilie AS, Sampaio-Baptista C, O'Shea J, Woolrich M, Smith SM, Filippini N, Near J, Johansen-Berg H (2014) Local GABA concentration is related to network-level resting functional connectivity. Elife 3:e01465. CrossRef
Tomasi D, Volkow ND (2012) Aging and functional brain networks. Mol Psychiatry 17:471, 549-458. CrossRef Medline

Tomassini V, Jbabdi S, Klein JC, Behrens TE, Pozzilli C, Matthews PM, Rushworth MF, Johansen-Berg H (2007) Diffusion-weighted imaging tractography-based parcellation of the human lateral premotor cortex identifies dorsal and ventral subregions with anatomical and functional specializations. J Neurosci 27:10259-10269. CrossRef Medline

Tremblay S, Lafleur LP, Proulx S, Beaulé V, Latulipe-Loiselle A, Doyon J, Marjańska M, Théoret H (2016) The effects of bi-hemispheric M1-M1 transcranial direct current stimulation on primary motor cortex neurophysiology and metabolite concentration. Restor Neurol Neurosci 34: 587-602. CrossRef Medline

Van Dijk KR, Sabuncu MR, Buckner RL (2012) The influence of head motion on intrinsic functional connectivity MRI. Neuroimage 59:431-438. CrossRef Medline

Venkatakrishnan A, Sandrini M (2012) Combining transcranial direct current stimulation and neuroimaging: novel insights in understanding neuroplasticity. J Neurophysiol 107:1-4. CrossRef Medline

Verbeke G, Molenberghs G (2000) Linear mixed models for longitudinal data. New York: Springer.

Watson D, Clark LA, Tellegen A (1988) Development and validation of brief measures of positive and negative affect: the PANAS scales. J Pers Soc Psychol 54:1063-1070. CrossRef Medline

Wilke S, List J, Mekle R, Lindenberg R, Bukowski M, Ott S, Schubert F, Ittermann B, Flöel A (2017) No effect of anodal transcranial direct current stimulation on gamma-aminobutyric acid levels in patients with recurrent mild traumatic brain injury. J Neurotrauma 34:281-290. CrossRef Medline

Woods AJ, Antal A, Bikson M, Boggio PS, Brunoni AR, Celnik P, Cohen LG, Fregni F, Herrmann CS, Kappenman ES, Knotkova H, Liebetanz D, Miniussi C, Miranda PC, Paulus W, Priori A, Reato D, Stagg C, Wenderoth N, Nitsche MA (2016) A technical guide to tDCS, and related non-invasive brain stimulation tools. Clin Neurophysiol 127:1031-1048. CrossRef Medline

Yousry TA, Schmid UD, Alkadhi H, Schmidt D, Peraud A, Buettner A, Winkler P (1997) Localization of the motor hand area to a knob on the precentral gyrus. A new landmark. Brain 120:141-157. CrossRef Medline 